\title{
Time-Enhancement Curve of Four-Dimensional Computed Tomography Predicts Aneurysm Enlargement with Type-II Endoleak after Endovascular Aneurysm Repair
}

\author{
Yunosuke Nishihara, MD, $\mathrm{PhD}^{1}$ Kota Mitsui, $\mathrm{RT}^{1} \quad$ Shinya Azama, $\mathrm{MD}^{1}$ Daisuke Okamoto, MD, $\mathrm{PhD}^{1}$ \\ Manabu Sato, MD, PhD² Kozo Naito, MD, PhD² Hitoshi Aibe, MD \\ ${ }^{1}$ Department of Radiology, Saga-Ken Medical Centre, Koseikan, \\ Saga, Japan \\ 2 Department of Cardiovascular Surgery, Saga-Ken Medical Centre, \\ Koseikan, Saga, Japan \\ Address for correspondence Yunosuke Nishihara, MD, Department of \\ Radiology, Saga-Ken Medical Centre Koseikan, 400 Nakabaru, \\ Kase, Saga 840-8571, Japan (e-mail: yunosuke248ra@gmail.com).
}

AORTA 2020;8:29-34.

\begin{abstract}
Keywords

- endovascular aneurysm repair

- Type-II endoleak

- computed tomography

- time-enhancement curve

Objective We investigated the hemodynamic features of Type-II endoleaks after endovascular aneurysm repair (EVAR) using four-dimensional (4D) computed tomography $(C T)$ to identify patients with aneurysm enlargement.

Methods During a 13-month period (January 2017-January 2018) at our institution, we performed 4D-CT examinations in 13 patients after EVAR because of suspected Type-II endoleaks. Three patients were excluded from the study because of other endoleaks or absence of detectable endoleaks. The remaining 10 patients were divided into two groups: enlargement group $(n=4)$, in which the aneurysm volume increased, and stable group $(n=6)$, in which the aneurysm remained stable or shrank. A CT scanner and three-dimensional workstation were used. All images were obtained using a consistent protocol (22 phase scans using the test bolus tracking method). We analyzed the hemodynamics of the endoleak cavity (EC) relative to those of the aorta and evaluated the time-enhancement curves (TECs) using measurement protocols. The strengths of correlations between these factors in the two groups were analyzed statistically.

Results TECs in the enlargement group showed a more gradual curve, and the upslope, the gradient of TEC in the ascending phase and the upslope index were significantly more gradual than those in the stable group $(p=0.0247,0.0243)$. The EC washout and the EC washout index were also more gradual than in the stable group's $(p=0.019,0.019)$. The enhancement duration was longer in the former than in the latter $(80 \%, p=0.0195 ; 70 \%, p=0.0159 ; 60 \%, p=0.0159)$. The CT number in the equilibrium phase was larger in the enlargement group than in the stable group $(p=0.019)$.

Conclusion The 4D-CT is useful for predicting aneurysm enlargement with Type-II endoleaks after EVAR.
\end{abstract}

received

July 30, 2018

accepted after revision

December 18, 2019
DOI https://doi.org/

10.1055/s-0040-1702144. ISSN 2325-4637.
Copyright $\odot 2020$ by Thieme Medical Publishers, Inc., 333 Seventh Avenue, New York, NY 10001, USA. Tel: +1(212) 760-0888.
License terms

(c) (i) 


\section{Introduction}

The most frequent mechanism of failure after endovascular aneurysm repair (EVAR) is the occurrence of an endoleak. ${ }^{1,2}$ Most endoleaks are Type II, defined as retrograde flow into the aneurysmal sac, most commonly from the patent inferior mesenteric artery (IMA), and/or lumbar arteries (LA).

The clinical significance of persistent Type-II endoleaks that last more than 6 months has not been defined. Although Types I and III endoleaks require immediate intervention, ${ }^{1,3}$ the optimal treatment for persistent Type-II endoleaks is controversial, ${ }^{4}$ even though aneurysm growth occurs in approximately $50 \%$ of such patients. ${ }^{5}$ It is generally accepted that persistent Type-II endoleaks associated with aneurysm enlargement require aggressive management, because aneurysm enlargement is the most important predictor of aneurysm rupture. ${ }^{6}$ It would therefore be beneficial to understand which imaging findings related to Type-II endoleaks are associated with the future need for intervention. To our knowledge, only a few small studies have examined a limited number of Type-II endoleak features and patient characteristics in an attempt to predict which Type-II endoleaks will progress, necessitating intervention, and which will not. ${ }^{7-10}$

Conventional biphasic computed tomography (CT) angiography, usually consisting of arterial and venous phases, is the current standard method for pre- and postoperative imaging evaluations of abdominal aortic aneurysm. The recent introduction of four-dimensional computed tomography (4D-CT) has allowed the assessment of temporal enhancement patterns in the endograft, aneurysm sac, and adjacent aortic branches with a high temporal resolution. We designed the present study to reveal the hemodynamic features of Type-II endoleaks after EVAR using 4D-CT, to identify patients with aneurysm enlargement.

\section{Materials and Methods}

\section{Patient Population}

This was a case-control single-institutional study approved by our institutional review board. We obtained informed consent from all of the patients included. We performed 4D-CT examinations in 13 patients after EVAR with suspected Type-II endoleaks during a 13-month period (January 2017-January 2018) at Saga-Ken Medical Centre Koseikan (-Fig. 1). After EVAR, all of the patients underwent follow-up CT scans at the 1-week, 6- and 12-month follow-up points and annually thereafter in our institution. Three patients were excluded from this study because of Type-III endoleak $(n=1)$ and absence of detectable endoleaks (Type-V endoleak; $n=2$ ). The remaining 10 patients were divided into two groups: enlargement group and stable group. Four patients were in the enlargement group where the aneurysm volume increased by $>2 \%$. In all these cases, the enlargement was confirmed at the next follow-up CT examinations. Six patients were in the stable group where the aneurysm volume remained the same or was reduced at the next follow-up CT examinations. This group included two cases in which endoleaks had disappeared by the time of the next examination.

\section{Image Acquisition}

All CT studies were performed with a 320-row CT scanner (Aquilion ONE; Canon Medical Systems, Tochigi, Japan). Scanning parameters were kept as consistent as possible. The scanning parameters for 4D-CT were: acquisition, 22 phases; scan mode, volume; tube voltage, $100 \mathrm{kVp}$; tube current, $100 \mathrm{~mA}$; rotation time, 0.5 second/rotation; collimation, $0.5 \mathrm{~mm} \times 320$; and $\mathrm{CTDI}_{\mathrm{vol}}, 70.1 \mathrm{mGy}$. The radiation dose is almost the same as that of conventional follow-up CT examination consists of three times scanning (precontrast image and two phase contrast images). We injected contrast medium using the test bolus tracking (TBT) method. ${ }^{11}$ Iopamidol was injected via the right antecubital vein at 2.5 to $4.5 \mathrm{~mL} / \mathrm{s}$ (fractional dose: $24 \mathrm{mg} / \mathrm{kg} / \mathrm{s}$ ) for 3 seconds, immediately followed by a saline flush injected for 5 seconds as a test bolus injection. After an interval of 15 seconds, the main bolus composed of contrast medium for 10 seconds followed by a saline flush for 5 seconds was injected. The TBT method made it possible to start scanning just before the main bolus arrived at the aorta. Equilibrium phase images were also acquired 90 seconds after the arrival

\begin{tabular}{|c|c|}
\hline \multicolumn{2}{|c|}{$\begin{array}{l}13 \text { patients who underwent 4DCTs because of suspected Type II endoleak } \\
\text { after EVAR during a 13-month period (Jan } 2017 \text { to Jan 2018) }\end{array}$} \\
\hline \multicolumn{2}{|c|}{ Follow-up CT after EVAR at 1 -week, 6- and 12-month and annually thereafte } \\
\hline \multicolumn{2}{|c|}{\begin{tabular}{|l|}
3 patients were excluded for the reasons of \\
2 cases: absence of detectable endoleak (Type V endoleak) \\
1 case: Type III endoleak \\
\end{tabular}} \\
\hline \multicolumn{2}{|l|}{ The 10 patients were included in the study } \\
\hline $\begin{array}{l}\text { Enlargement group } \\
4 \text { patients with sac enlargement }(>2 \% \\
\text { increase in volume) }\end{array}$ & $\begin{array}{l}\text { Stable group } \\
6 \text { patients with stable sac volume or } \\
\text { shrinkage }\end{array}$ \\
\hline
\end{tabular}

Fig. 1 Flowchart of study profile. 4D, four-dimensional; CT, computed tomography; EVAR, endovascular aneurysm repair. 
time at the aorta. Acquired images were reconstructed and archived as $0.5-\mathrm{mm}$-thick sections with no overlap.

\section{Image Interpretation}

All CT examinations in 10 patients with Type-II endoleaks were retrospectively measured in greater detail to evaluate the TEC of the endoleak cavity (EC) and that of the aorta. Measurements were performed by one observer (K.M. with 10 years' experience analyzing vascular CT images). Measurements of the TEC and aneurysm volumetry were performed using commercial image postprocessing software (Ziostation2 ver. 2.4.2.3, Ziosoft, Tokyo). The TEC of the aorta was measured by positioning a region of interest in the aorta at the level of the ostial superior mesenteric artery. Measurements of the TEC were performed by positioning a region of interest within the $\mathrm{EC}$ at a representative section away from feeding/draining arteries to avoid any hemodynamic influence of side-branch vessels. We evaluated the TEC using the measurement protocol shown in -Fig. 2. We also recorded arrival time; arrival delay time, the difference between the arrival time at the EC and at the aorta; time to peak enhancement, the difference between the CT peak time and the arrival time; maximal CT number, CT peak; and the CT number in equilibrium phase, 90 seconds after the arrival time at the aorta. We measured the gradient of the curve in the ascending phase as the upslope, and the gradient of the curve in descending phase as the EC washout. Peak-to-peak time was defined as the difference between the CT peak time at the EC and the aorta. The duration of contrast enhancement achieved within $80 \%$ (70 and $60 \%$ ) of the peak was defined as $80 \%$ (70 and 60\%) enhancement duration. We also measured the area under the curve (AUC) and the area under the TEC. We defined the upslope index and EC washout index to calibrate circulatory conditions. The upslope index was defined as the upslope of the EC divided by the upslope of the aorta. The EC washout index was defined as the EC washout divided by that of the aorta.

\section{Statistical Analysis}

We performed univariate analyses for comparisons of the enlargement group and the stable group. Continuous variables were expressed as medians (interquartile range [IQR]); these were analyzed with the Mann-Whitney $U$-test for unpaired comparisons (-Table 1). All statistical analyses were performed with EZR (Saitama Medical Center, Jichi Medical University, Saitama, Japan), which is a graphical user interface for R (The R Foundation for Statistical Computing, Vienna, Austria). ${ }^{12}$ More precisely, EZR is a modified version of $\mathrm{R}$ commander (ver. 1.33) designed to add statistical functions frequently used in biostatistics. Findings were considered significant when the $p$-value was $<0.05$.

\section{Results}

\section{Time-Enhancement Curve Characteristics}

There were many significant between-group differences in certain aspects of the TEC characteristics (-Table 1). There were no significant differences between the two groups in the arrival time, arrival delay time, time to peak enhancement, and CT peak. The upslope was significantly more gradual in the enlargement group than in the stable group: $7 \mathrm{HU} / \mathrm{s}$, IQR, 7 to 8 versus $24 \mathrm{HU} / \mathrm{s}, \mathrm{IQR}, 17$ to $30 \mathrm{HU} / \mathrm{s}(p=0.0247)$. The upslope index was significantly smaller in the enlargement group than in the stable group: $0.21, \mathrm{IQR}, 0.18$ to 0.26 versus 0.72 , IQR, 0.49 to 1.04 ( $p=0.0243$ ). EC washout was also significantly more gradual in the enlargement group than in the stable group: $1.96 \mathrm{HU} / \mathrm{s}, \mathrm{IQR}, 1.31$ to 3.38 versus $16.6 \mathrm{HU} / \mathrm{s}, \mathrm{IQR}, 14.6$ to 21.2 $\mathrm{HU} / \mathrm{s}(p=0.019)$. The EC washout index was also significantly smaller in the enlargement group than in the stable group: $0.07, \mathrm{IQR}, 0.05$ to 0.12 versus 0.70 , IQR, 0.57 to $0.82(p=0.019$ ). There were no significant differences between the two groups in the peak to peak time. The enhancement duration time was significantly longer in the enlargement group than in the stable group at each percentage: $80 \%$; 14.8 seconds, IQR, 13.7 to 16.1 versus 7.4 seconds; IQR, 6.1 to 7.4 seconds ( $p=0.0195$ ); 70\%; 20.7 seconds, IQR, 18.9 to 22.2 versus 8.4 seconds; IQR, 8.3 to

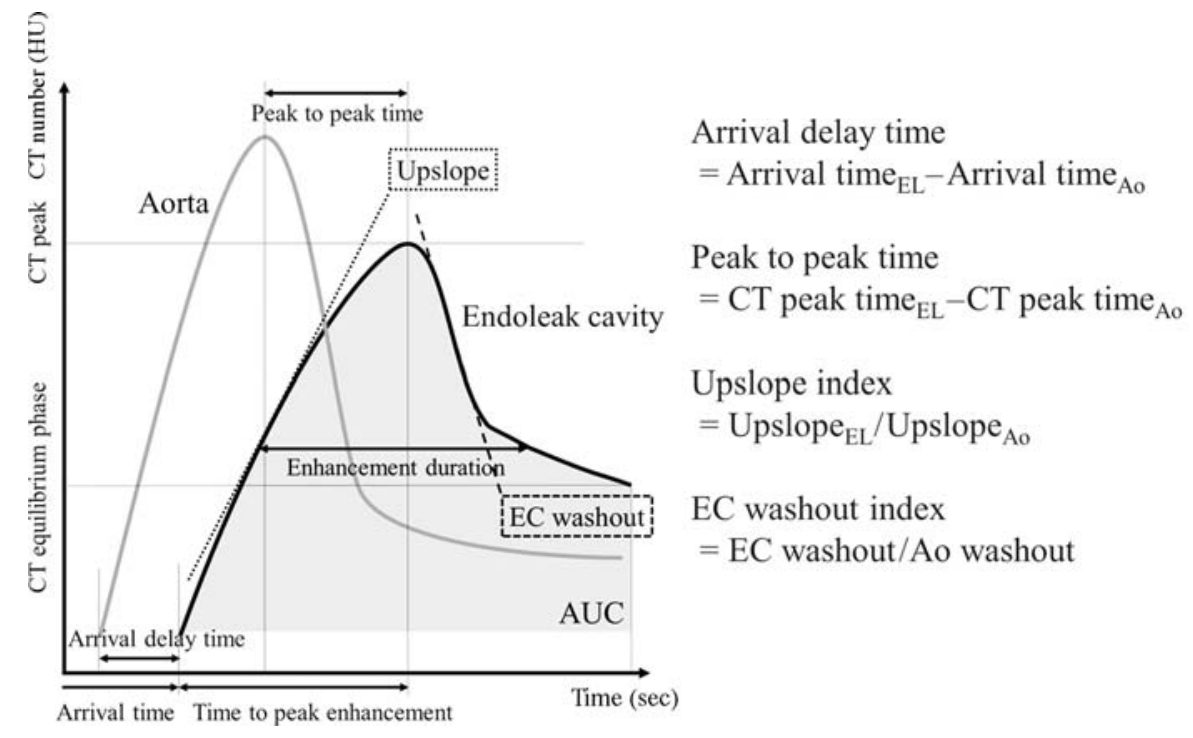

Fig. 2 Measurement protocol of time-enhancement curve. AUC, area under curve; CT, computed tomography; EC, endoleak cavity. 
Table 1 Time-enhancement curve characteristics comparisons of enlargement and stable groups

\begin{tabular}{|l|l|l|l|}
\hline Parameter & $\begin{array}{l}\text { Enlargement group } \\
\boldsymbol{n}=\mathbf{4}\end{array}$ & $\begin{array}{l}\text { Stable group } \\
\boldsymbol{n}=\mathbf{6}\end{array}$ & $\boldsymbol{p}$-Value \\
\hline Arrival time (s) & $15(13-22)$ & $9(7-10)$ & 0.106 \\
\hline Arrival delay time (s) & $13(12-16)$ & $7(6-10)$ & 0.133 \\
\hline Time to peak enhancement (s) & $17(14-19)$ & $12(11-14)$ & 0.234 \\
\hline CT peak (HU) & $192(130-262)$ & $265(227-319)$ & 0.352 \\
\hline Upslope (HU/s) & $7(7-8)$ & $24(17-30)$ & $\mathbf{0 . 0 2 4 7}$ \\
\hline Upslope index & $0.21(0.18-0.26)$ & $0.72(0.49-1.04)$ & $\mathbf{0 . 0 2 4 3}$ \\
\hline EC washout (HU/s) & $1.96(1.31-3.38)$ & $16.6(14.6-21.2)$ & $\mathbf{0 . 0 1 9}$ \\
\hline EC washout index & $0.07(0.05-0.12)$ & $0.70(0.57-0.82)$ & $\mathbf{0 . 0 1 9}$ \\
\hline Peak to peak time (s) & $17(16-19)$ & $5(4-6)$ & 0.108 \\
\hline $80 \%$ enhancement duration (s) & $14.8(13.7-16.1)$ & $7.4(6.1-7.4)$ & $\mathbf{0 . 0 1 9 5}$ \\
\hline $70 \%$ enhancement duration (s) & $20.7(18.9-22.2)$ & $8.4(8.3-8.6)$ & $\mathbf{0 . 0 1 5 9}$ \\
\hline $60 \%$ enhancement duration (s) & $29.9(27.3-33.0)$ & $10.6(10.1-11.7)$ & $\mathbf{0 . 0 1 5 9}$ \\
\hline AUC (HU $\times$ s) & $11,909(11,642-12,117)$ & $12,815(9,766-15,661)$ & 1 \\
\hline CT equilibrium phase (HU) & $90(75-103)$ & $27(22-39)$ & $\mathbf{0 . 0 1 9}$ \\
\hline
\end{tabular}

Abbreviations: AUC, area under curve; CT, computed tomography; EC, endoleak cavity; TEC, time-enhancement curve. Note: all data are medians. Numbers in parentheses are the interquartile range (lower quartile[Q1]-upper quartile[Q3]).

8.6 seconds ( $p=0.0159$ ); $60 \%$; 29.9 seconds, IQR, 27.3 to 33.0 versus 10.6 seconds; IQR, 10.1 to 11.7 seconds $(p=0.0159)$. There were no significant differences in AUC between the two groups. The CT equilibrium phase was significantly higher in the enlargement group than in the stable group: $90 \mathrm{HU}, \mathrm{IQR}, 75$ to 103 versus $27 \mathrm{HU}, \mathrm{IQR}, 22$ to $39 \mathrm{HU}(p=0.019)$.

\section{Discussion}

The need for vigilant surveillance with optimized CT angiography protocols and image postprocessing methods is strongly supported by evidence of aneurysm rupture in patients with persistent Type-II endoleaks. ${ }^{13}$ The most important parameter for indicating nonrupture in a patient with Type-II endoleak is a stable aneurysm sac volume on CT angiography images, and previous work has shown no significant differences in clinical characteristics (e.g., sex, smoking history, hypertension, need for anticoagulation, aneurysm diameter, and type of endograft used) between patients with enlarging aneurysms and those with either shrinking or stable aneurysms. ${ }^{14}$ Other conventional CT angiography studies have revealed several indicators of aneurysm enlargement. Demehri et al ${ }^{15}$ showed that the EC volume in patients with aneurysm enlargement was significantly larger than that in patients with stable or shrinking aneurysms. By analyzing delayed acquisition CT images, Timaran et $\mathrm{al}^{8}$ demonstrated that the maximum diameter of an EC can be used to accurately indicate sac enlargement. Previous studies ${ }^{8,16}$ have also showed that the median number of feeding and/or draining arteries was greater in patients with enlarged aneurysms than in patients with stable or reduced aneurysms. Müller-Wille et al ${ }^{16}$ categorized Type-II endoleaks into more detailed subtypes, and they stated that the strongest indicators for aneurysm sac enlargement were complex
IMA-LA Type-II endoleaks and the diameter of the largest feeding and/or draining artery.

We analyzed the TEC of the EC and the aorta in 4D-CT images to evaluate the hemodynamic characteristics. We found that patients with sac enlargement had longer enhancement duration, a more gradual upslope and gradual EC washout and a higher CT equilibrium phase (-Fig. 3). That is, the TEC in patients with sac enlargement became more gradual. Bargellini et $\mathrm{al}^{7}$ showed that a longer washout time (>520 seconds) was the only independent predictor of aneurysm sac enlargement in patients with persistent Type-II endoleaks identified by contrast-enhanced ultrasound scanning. Demehri et al $^{15}$ also reported four CT examinations in which Type-II endoleaks were observed and measured only in the delayed phase and demonstrated aneurysm enlargement. Their arguments are consistent with the results of our TEC analyses. That is, the TEC of the EC measured only in the delayed phase or with a longer washout time lay on a gradual curve with a gradual upslope and a gradual EC washout, and larger CT number at the equilibrium phase correlated with subsequent aneurysm enlargement. Dias et $\mathrm{al}^{9}$ measured the intra-aneurysm sac pressure directly using tip-pressure sensors through direct translumbar puncture. In that study, Type-II endoleaks in shrinking aneurysms had a lower intrasac pressure than expanding or stable aneurysms. We presume that in patients with aneurysm shrinkage, a lower intrasac pressure induces a shorter arrival delay time and a shorter peak to peak time, which cause a steep TEC in 4D-CT. However, the arrival delay time and the peak to peak time in the stable group tended to be short overall than those in the enlargement group, but we could not prove statistical difference. We believe that a large cohort study will demonstrate more certain correlations. 

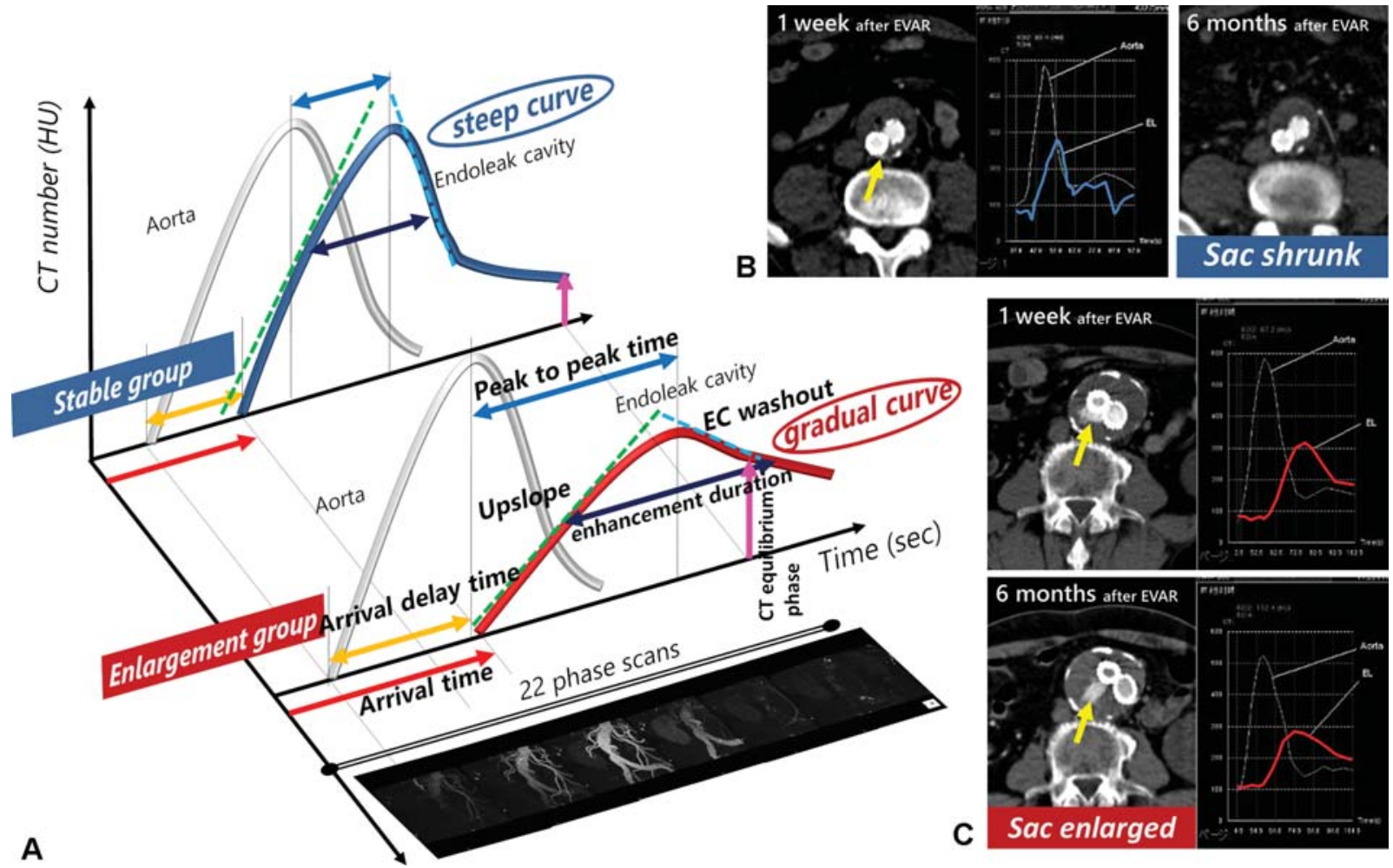

ig. 3 (A) Schema of the time-enhancement curve (TEC) of a Type II endoleak. (B and C) Two actual cases. (B) A 71-year-old male underwent endovascular aneurysm repair (EVAR), and four-dimensional computed tomography (4D-CT) after 1 week revealed a tiny Type-Il endoleak (yellow arrow). The TEC of the endoleak was steep (blue curve). Six months after EVAR, the Type-Il endoleak had disappeared and the aneurysm sac had shrunk (30-23 cC). (C) An 85-year-old male underwent EVAR and 4D-CT after 1 week revealed a Type-Il endoleak (yellow arrow). The TEC of the endoleak was gradual (red curve). Six months after EVAR, a persistent Type-II endoleak was found and the aneurysm sac was enlarged (69-79 cc). The TEC pattern was similar to that at 1 week after EVAR.

Lehmkuhl et al ${ }^{17}$ revealed that the peak enhancement of an endoleak is different than that of the aorta and that endoleaks may not be adequately evaluated using conventional biphasic CT protocols, suggesting that $4 \mathrm{D}-\mathrm{CT}$ increases the endoleak detection rate. We consider that 4D-CT and TEC analyses can differentiate other types of endoleaks, detect small endoleaks, and furthermore can predict sac enlargement. Our search of the relevant literature did not identify any report suggesting the importance of the TEC for $4 \mathrm{D}-\mathrm{CT}$ as an indicator of aneurysm sac enlargement.

\section{Limitations}

Several limitations of our study should be considered. First, all of the patients in this study were diagnosed based on $4 \mathrm{D}-\mathrm{CT}$ images; they did not undergo conventional angiography for evaluation of endoleaks which some researchers consider essential for proper classification of all endoleaks. ${ }^{2,4}$ Second, we did not validate the accuracy of the volumetry using a phantom model of endoleaks. One observer obtained all of the measurements, and we were unable to determine the interobserver measurement variability. Finally, our patient series was somewhat small, thus preventing us from exploring other clinical applications of TEC analyses.

\section{Conclusion}

The results of the present study demonstrated that $4 \mathrm{D}-\mathrm{CT}$ is useful for predicting aneurysm enlargement with Type-II endoleaks after EVAR. TEC characteristics should be considered when interpreting follow-up CT examinations of patients with Type-II endoleaks, because this will provide an important guide for monitoring and determining the need for additional interventions in these patients.

Funding

None.

Conflict of Interest

The authors declare no conflict of interest related to this article.

\section{Acknowledgments}

None.

\section{References}

1 Veith FJ, Baum RA, Ohki T, et al. Nature and significance of endoleaks and endotension: summary of opinions expressed at an international conference. J Vasc Surg 2002;35(05): 1029-1035 
2 Rhee SJ, Ohki T, Veith FJ, Kurvers H. Current status of management of type II endoleaks after endovascular repair of abdominal aortic aneurysms. Ann Vasc Surg 2003;17(03):335-344

3 van Marrewijk C, Buth J, Harris PL, Norgren L, Nevelsteen A, Wyatt MG. Significance of endoleaks after endovascular repair of abdominal aortic aneurysms: The EUROSTAR experience. J Vasc Surg 2002;35(03):461-473

4 Baum RA, Carpenter JP, Golden MA, et al. Treatment of type 2 endoleaks after endovascular repair of abdominal aortic aneurysms: comparison of transarterial and translumbar techniques. J Vasc Surg 2002;35(01):23-29

5 van Marrewijk CJ, Fransen G, Laheij RJ, Harris PL, Buth J; EUROSTAR Collaborators. Is a type II endoleak after EVAR a harbinger of risk? Causes and outcome of open conversion and aneurysm rupture during follow-up. Eur J Vasc Endovasc Surg 2004;27 (02):128-137

6 Bernhard VM, Mitchell RS, Matsumura JS, et al. Ruptured abdominal aortic aneurysm after endovascular repair. J Vasc Surg 2002; 35(06):1155-1162

7 Bargellini I, Napoli V, Petruzzi P, et al. Type II lumbar endoleaks: hemodynamic differentiation by contrast-enhanced ultrasound scanning and influence on aneurysm enlargement after endovascular aneurysm repair. J Vasc Surg 2005;41(01):10-18

8 Timaran $\mathrm{CH}$, Ohki T, Rhee SJ, et al. Predicting aneurysm enlargement in patients with persistent type II endoleaks. J Vasc Surg 2004;39(06):1157-1162

9 Dias NV, Ivancev K, Resch TA, Malina M, Sonesson B. Endoleaks after endovascular aneurysm repair lead to nonuniform intraaneurysm sac pressure. J Vasc Surg 2007;46(02):197-203
10 Keedy AW, Yeh BM, Kohr JR, Hiramoto JS, Schneider DB, Breiman RS. Evaluation of potential outcome predictors in type II Endoleak: a retrospective study with CT angiography feature analysis. AJR Am J Roentgenol 2011;197(01):234-240

11 Yamaguchi T, Takahashi D. [Development of test bolus tracking method and usefulness in coronary CT angiography]. Nippon Hoshasen Gijutsu Gakkai Zasshi 2009;65(08):1032-1040

12 Kanda Y. Investigation of the freely available easy-to-use software 'EZR' for medical statistics. Bone Marrow Transplant 2013;48(03): 452-458

13 Ohki T, Veith FJ, Shaw P, et al. Increasing incidence of midterm and long-term complications after endovascular graft repair of abdominal aortic aneurysms: a note of caution based on a 9-year experience. Ann Surg 2001;234(03):323-334, discussion 334-335

14 Sarac TP, Gibbons C, Vargas L, et al. Long-term follow-up of type II endoleak embolization reveals the need for close surveillance.J Vasc Surg 2012;55(01):33-40

15 Demehri S, Signorelli J, Kumamaru KK, et al. Volumetric quantification of type II endoleaks: an indicator for aneurysm sac growth following endovascular abdominal aortic aneurysm repair. Radiology 2014;271(01):282-290

16 Müller-Wille R, Schötz S, Zeman F, et al. CT features of early type II endoleaks after endovascular repair of abdominal aortic aneurysms help predict aneurysm sac enlargement. Radiology 2015; 274(03):906-916

17 Lehmkuhl L, Andres C, Lücke C, et al. Dynamic CT angiography after abdominal aortic endovascular aneurysm repair: influence of enhancement patterns and optimal bolus timing on endoleak detection. Radiology 2013;268(03):890-899 